\title{
Editorial
}

Tran Quoc Khanh and Cornelius Neumann*

\section{Illumination optics for indoor lighting, automotive and street lighting}

https://doi.org/10.1515/aot-2019-0007

In the 20th Century, illumination optics systems were developed based on the concepts of reflectors, prisms and Fresnel optics for incandescent tungsten and discharge lamps of relatively extensive geometrical dimensions. Luminous intensity distributions and spectral as well as color properties were generally constant over the time of use. Therefore, it was not possible to develop smart lighting systems with variable spectral, geometrical and colorimetric features depending on application context (e.g. school, hospital, office, small or main street in a city), on the time of the day and on weather conditions.

In the last 12 years, LED technology has been developing at a very fast pace. In the period from 2004 until 2016, the focus of lighting technology and lighting companies was on the elaboration of stable and efficient luminaire systems. Several new concepts of free-form reflector optics, highly efficient lens systems with cost-optimized manufacturing steps and new optical materials (e.g. optically transparent plastic materials, coatings, silicone) arose. The resulting new indoor and outdoor lighting systems exhibited high luminous efficiencies in the order of $145-160 \mathrm{~lm} / \mathrm{W}$ as well as increased mechanical and optical stability.

In the last 3-4 years, the philosophy and the concepts of lighting design in the lighting industry have achieved a new quality level. The concepts of human centric lighting that represent a deep knowledge on the impact of light on human behavior (alertness, sleep quality, mood, activity, well-being) combined with the concepts of Internet-based lighting control and cloud-based data processing applying machine learning and neuronal network methods will help lighting technology (in general) and illumination optics (in particular) achieve a new development stage.

*Corresponding author: Cornelius Neumann, Karlsruher Institut fur Technologie, Lichttechnisches Institut, Engesser Str. 13, Karlsruhe, Germany, e-mail: cornelius.neumann@kit.edu

Tran Quoc Khanh: Technische Universität Darmstadt, Department of Electrical Engineering and Information Technology, Fraunhoferstr. 4, 64283 Darmstadt, Germany

www.degruyter.com/aot

(C) 2019 THOSS Media and De Gruyter
Human centric lighting concepts can be efficiently implemented to achieve their strongest impact only in the context of "dynamic lighting". "Dynamic lighting” means the use of variable light source spectra with more or less non-visually stimulating spectral content in the range between $400 \mathrm{~nm}$ and $550 \mathrm{~nm}$, with variable luminous intensity distributions over the time of the day and with a dependence on weather conditions and lighting applications. Consequently, the following aspects of illumination optics design will become essential in the future:

(a) Details of the spectral and colorimetric distributions of the light from the light source (LED component or LED module) over the entire emission angle range will be necessary in order to design the optics to achieve a uniform white light distribution at a selected color temperature;

(b) A high-quality optics will be indispensable in order to mix the optical radiations from the different LED groups with different spectral distributions in order to obtain the desired polychromatic light beam with well-defined spectra and luminous intensity distributions. Colorimetric uniformity of the mixed light beam shall be ensured over the entire angular emission range and over the whole light emitting area;

(c) Photobiologically negative effects of optical radiation (e.g. glare and blue light hazard) should be limited by the design of suitable illumination optics.

Concepts and results of illumination optics design will be described in the present volume according to the abovementioned principles with contributions from internationally well-known experts working in the field.

During the last decade LED and active lighting technology dramatically changed the way we drive at night. Signal lamps define the night time styling appearance of modern cars, while adaptive driving beams guarantee a better illumination of the road ahead of us.

For the next generation of vehicles, several technologies for high resolution headlamps are on their way to serial application.

High resolution in the far field can help to illuminate the traffic scene at night to detect potential dangers 
without glaring other traffic participants. However, there will be an optimum in the number of pixels.

In the near field, high-resolution projection can be used for communication between the car and the driver (or other traffic participants). For this humanmachine interface, one needs to define the "semantics of light”, signals for an intuitively understandable communication.

Finally, new lighting systems have to be tested under reproducible conditions. Night-time driving simulation would be an appropriate environment for such testing, but at the moment, only situations without (self-) glaring can be monitored on the screen in a realistic manner. Future simulation have to represent realistic glare situations. For this, new approaches in projection technologies have to be investigated.

So, vision, glare and communication are actual pace makers of automotive head lamp technology. For these topics, you will find interesting contributions from automotive lighting experts in this issue.

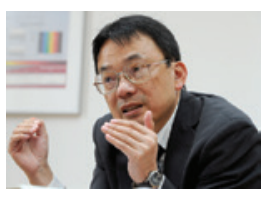

\section{Tran Quoc Khanh}

Technische Universität Darmstadt

Department of Electrical Engineering and

Information Technology

Fraunhoferstr. 4, 64283 Darmstadt, Germany

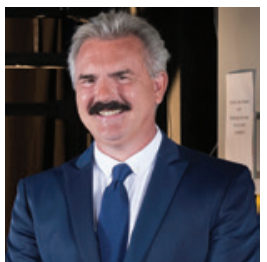

Cornelius Neumann

Karlsruher Institut fur Technologie

Lichttechnisches Institut, Engesser Str. 13

Karlsruhe, Germany

cornelius.neumann@kit.edu

Cornelius Neumann studied Physics and Philosophy at the University of Bielefeld, Germany. After his PhD, he worked for the automotive supplier Hella in the advanced development for automotive lighting. During his time at Hella he was responsible for signal lighting, LED application and acted as a director of the L-LAB, a laboratory for lighting and mechatronics in public private partnership with the University of Paderborn, Germany. In 2009, he became Professor for Optical Technologies in Automotive and General Lighting and one of the two directors of the Light Technology Institute at the Karlsruhe Institute of Technology, Germany.

Tran Quoc Khanh wrote his PhD thesis on the spectroscopy of UV-VIS radiation sources in 1989. After 17 years of industrial experience, he completed his Lecture Qualified Thesis (habilitation) on color appearance and visual performance and started his current work as a professor for lighting technology and solid state lighting at the Technische Universität Darmstadt. He is conducting research and development projects on LED lighting technology. He is also the chairman of the International Symposium for Automotive Lighting (ISAL). He is the author of several books and scientific articles and inventor of patents on lighting technology and related subjects. 